\title{
Teor e composição química do óleo essencial e crescimento vegetativo de Aloysia triphylla em diferentes espaçamentos e épocas de colheita
}

\author{
Dalva Paulus ${ }^{1}$, Raquel Valmorbida², Ezequiel Toffoli ${ }^{3}$, Gilmar Antônio Nava ${ }^{4}$, Eloi Paulus ${ }^{5}$
}

\section{RESUMO}

Objetivou-se, com este trabalho, avaliar a produção de biomassa, o teor e os componentes do óleo essencial de Aloysia triphylla, em função de diferentes espaçamentos de plantas e épocas de colheita. O experimento foi conduzido na área experimental da Universidade Tecnológica Federal do Paraná - Campus Dois Vizinhos, no período de março de 2010 a janeiro de 2012. O delineamento experimental utilizado foi o de blocos ao acaso, com três repetições, em esquema fatorial ( 3 x 11), parcelas subdivididas, sendo alocados na parcela três espaçamentos de plantio (1,0 x 1,0 m, 1,0 x 0,80 $\mathrm{m}, 1,0 \times 0,60 \mathrm{~m}$, entre linhas e entre plantas, respectivamente) e, nas subparcelas, 11 épocas de colheita (outubro/2010, novembro/2010, dezembro/2010, janeiro/2011, fevereiro/2011, março/2011, abril/2011, maio/2011, junho/2011, agosto/ 2011, setembro/2011). As características analisadas foram: altura de plantas, área foliar, biomassas fresca e seca, teor e composição química do óleo essencial. O óleo essencial foi extraído pela técnica de hidrodestilação, utilizando-se o aparelho de Clevenger, e analisado em cromatografia gasosa e espectrofotometria de massa (CG/EM). O espaçamento 1,0 x 1,0 m resultou em maior altura de plantas $(1,57 \mathrm{~m})$, área foliar $\left(4.460,3 \mathrm{~cm}^{2}\right)$, biomassas fresca $(1.266 \mathrm{~g}$ por planta) e seca (594 g por planta) e teor de óleo essencial (0,30\%). A colheita realizada no mês de fevereiro resultou em maior teor de óleo essencial $(0,45 \%)$. Os componentes químicos do óleo essencial variam com espaçamentos e épocas de colheita, sendo o maior teor de citral no espaçamento $1,0 \times 1,0 \mathrm{~m}$ e nos meses de setembro a abril.

Palavras-chave: Aloysia triphylla, densidade de plantio, sazonalidade.

\section{ABSTRACT \\ Content and constituents of essential oil of Aloysia triphylla in different row spaces and harvest times}

This study aimed to evaluate biomass production, content and components of the essential oil of Aloysia triphylla in different row spaces and harvest times. The experiment was conducted at the experimental area of Universidade Federal Tecnológica do Paraná - Campus Dois Vizinhos, from March 2010 to January 2012. The experimental design was a randomized block design with three replications in a factorial scheme $(3 \times 11)$, subdivided blocks allocated in three planting rows plot $(1.0 \times 1.0 \mathrm{~m}, 1.0 \times 0.80 \mathrm{~m}, 1 \times 0,60 \mathrm{~m}$ between rows and between plants, respectively) and on subplots, 11 harvest times (October/2010, November/2010, December/2010, January/2011, February/2011, March/2011, April/2011, May/2011, June/2011, August / 2011, September/2011). The characteristics assessed were: plant height, leaf area, fresh and dry matter, content and chemical composition of essential oil. The essential oil was extracted by

Recebido para publicação em 12/06/2012 e aprovado em 23/04/2013

${ }^{1}$ Engenheira-Agrônoma, Doutora. Departamento de Horticultura, Universidade Tecnológica Federal do Paraná, Campus Dois Vizinhos, Estrada para Boa Esperança, Km 04, Caixa Postal 157, 85660- 000, Dois Vizinhos, Paraná, Brasil. dalvaufsmdeutch@yahoo.com.br (autora para correspondência).

${ }^{2}$ Graduanda em Engenharia Florestal. Departamento de Horticultura, Universidade Tecnológica Federal do Paraná, Campus Dois Vizinhos, Estrada para Boa Esperança, Km 04, Caixa Postal 157, 85660- 000, Dois Vizinhos, Paraná, Brasil. raquelvalmorbida@yahoo.com.br (Bolsista de Iniciação Científica).

${ }^{3}$ Graduando em Engenharia Florestal. Departamento de Horticultura, Universidade Tecnológica Federal do Paraná, Campus Dois Vizinhos, Estrada para Boa Esperança, Km 04, Caixa Postal 157, 85660- 000, Dois Vizinhos, Paraná, Brasil. toffoliezequiel@ yahoo.com.br

${ }^{4}$ Engenheiro-Agrônomo, Doutor. Departamento de Horticultura, Universidade Tecnológica Federal do Paraná, Campus Dois Vizinhos, Estrada para Boa Esperança, Km 04, Caixa Postal 157, 85660- 000, Dois Vizinhos, Paraná, Brasil. gilmarnava@utfpr.edu.br

${ }^{5}$ Engenheiro Florestal, Mestre. Departamento de Solos, Universidade Federal de Santa Maria, Avenida Roraima, Faixa de Camobi, Km 9, 97105-900, Santa Maria, Rio grande do Sul, Brasil. epaulus2000@yahoo.com.br 
hydrodistillation technique, using a Clevenger apparatus and analyzed in a gas chromatography and mass spectrometry (GC / MS). The 1 x $1 \mathrm{~m}$ spacing resulted in greater height $(1.57 \mathrm{~m})$, leaf area $\left(4460.3 \mathrm{~cm}^{2}\right)$, fresh weight (1.266 g per plant), dry weight (594 g per plant) and content of essential oil (0.30\%). The harvest performed in February resulted in higher levels of essential oil $(0.45 \%)$. The chemical constituents of the essential oil vary with spacing and harvesting time. The highest content of citral was observed in plants cultivated at 1.0 x $1.0 \mathrm{~m}$ spacing and harvested from September to April.

Key words: Aloysia triphylla, planting density, seasonality.

\section{INTRODUÇÃO}

A espécie medicinal Aloysia triphylla (L’Hérit) Britton (Verbenaceae) é conhecida, popularmente, como limonete, ou cidrão. É uma espécie nativa da América do Sul e seu cultivo é feito no sul do Brasil. A planta possui porte arbustivo, com altura de 2 a $3 \mathrm{~m}$, ramificada e ereta. Tratase de uma erva adstringente, com propriedades aromáticas e rica em óleo volátil, que age como sedativo brando. Suas folhas têm grande importância medicinal, sendo empregadas no combate a resfriados febris, além de ser antiespasmódica, eupéptica, estimulante, calmante e tônica. Também é usada para tratamento contra afecções do coração, histeria e melancolia (Lorenzi \& Matos, 2008).

O componente químico de maior interesse, extraído da Aloysia triphylla, é o citral, substância que confere grande importância às indústrias farmacêutica e cosmética. Além do seu uso como perfume, o citral é empregado na síntese de ionona (perfume da violeta), beta-caroteno e vitamina A (Czepak \& Cruciol, 2003).

Figueiredo et al. (2004) identificaram, como constituintes majoritários do óleo essencial de Aloysia triphylla, geranial $(29,54 \%)$, neral $(27,01 \%)$, limoneno $(15,93 \%)$, acetato de geranila $(4,0 \%)$ e geraniol $(3,96 \%)$. Segundo os autores, esta espécie possui elevada quantidade de monoterpenos e baixa quantidade de sesquiterpenos.

O espaçamento entre plantas destaca-se como um dos fatores mais importantes do cultivo, pois influencia o crescimento e o rendimento final, que, por sua vez, depende da produtividade individual da planta e da população de plantas, mantida por unidade de área (Yadava, 2001). Além disso, a densidade de plantio no campo pode contribuir, positiva, ou negativamente, para a competição entre a cultura e as plantas invasoras por recursos como água, radiação e nutrientes (Monteiro et al., 2011).

A época de colheita também influencia o rendimento e composição do óleo essencial de plantas aromáticas. A biossíntese dos óleos essenciais é influenciada por fatores climáticos, como fotoperíodo, temperatura, umidade, precipitação e intensidade de radiação solar, que podem determinar a época ideal de colheita ou o local de cultivo, onde se poderão obter maiores quantidades de óleo essencial e do princípio ativo de interesse (Taveira et al., 2003).
No caso das espécies medicinais, a densidade de plantio e a época de colheita podem favorecer, ou não, a produtividade, em termos de biomassa e de princípios ativos de interesse para as indústrias farmacêutica e cosmética. O conhecimento prévio das condições de cultivo é de fundamental importância para estimular o aumento da produção dos componentes dos óleos essenciais (Lima et al., 2003) e, dessa forma, obter óleos essenciais com alta qualidade e melhores valores de mercado (Melo et al., 2011).

Marco et al. (2007) constataram que capim-citronela, na altura de corte de $15 \mathrm{~cm}$ e em densidades menores $(0,80$ x $0,80 \mathrm{~m})$, tem produção de óleo essencial maior $(40,02 \mathrm{~mL} /$ $\mathrm{kg}$ de massa seca) do que em densidades maiores de plantas $(0,50 \times 0,50 \mathrm{~m})$.

A influência da sazonalidade sobre a produção de óleo essencial de Alloysia triphila foi verificada por Brant et al. (2008), em Lavras, MG, onde os autores constataram que a melhor época de colheita foi de fevereiro a outubro, quando a produção de biomassa e de óleo extraído foram maiores. Ventrella \& Ming (2000) verificaram alterações no teor e na composição química do óleo essencial de erva-cidreira (Lippia alba Mill), em função das épocas de colheita e dos níveis de sombreamento. Os autores observaram que as colheitas realizadas em janeiro, quando a temperatura e a intensidade luminosa foram altas durante o desenvolvimento das plantas, a produção de matéria seca foliar, de 188, $23 \mathrm{~g} \mathrm{~m}^{2}$, e, a produção de óleo essencial, de $0,35 \mathrm{~mL} / 100 \mathrm{~g}$ de massa seca foram máximas, no cultivo a pleno sol.

Diante da escassez de informações, na literatura, sobre a Aloysia triphylla, e da importância de estudos sobre a influência do espaçamento de plantio e época de colheita das plantas, na produção de biomassa e teor de óleo essencial, a realização deste estudo se justifica, pois essas informações são de grande importância para o produtor de óleo essencial, no momento da colheita. Objetivou-se, com este trabalho, avaliar a produção de biomassa, o teor e os componentes químicos do óleo essencial de Aloysia triphylla, em função de diferentes espaçamentos e de épocas de colheita das plantas. 


\section{MATERIAL E MÉTODOS}

O trabalho foi realizado na área experimental da Universidade Tecnológica Federal do Paraná (UFTPR) Campus Dois Vizinhos, no período de março de 2010 a janeiro de 2012. O clima da região é classificado como Cfa subtropical úmido, sem estação seca definida e temperatura média do mês mais quente de $22^{\circ} \mathrm{C}$. O solo é do tipo Nitossolo Vermelho distroférrico (Bhering et al., 2008), que apresentou, em pré-plantio, as seguintes características químicas, na camada de 10 a $20 \mathrm{~cm}$ de profundidade: $\mathrm{pH}$ (água) $=5,7$; matéria orgânica $\left(\mathrm{g} \mathrm{dm}^{-3}\right)=41,55 ; \mathrm{P}, \mathrm{Cu}$, $\mathrm{Fe}, \mathrm{Zn}, \mathrm{Mn}\left(\mathrm{mg} \mathrm{dm}^{-3}\right): 31,29,6,67,19,67,7,89,197,26$, respectivamente; $\mathrm{Ca}, \mathrm{Mg}, \mathrm{K}, \mathrm{SB}, \mathrm{H}+\mathrm{Al}\left(\mathrm{cmol}_{\mathrm{c}} \mathrm{dm}^{-3}\right)=8,47$, $2,78,1,10,12,35,3,97$, respectivamente; $\mathrm{V}=75,67 \%$.

O delineamento experimental foi o de blocos ao acaso, com três repetições, em esquema fatorial ( 3 x 11) parcelas subdivididas, sendo alocados na parcela três espaçamentos de plantio ( 1,0 x 1,0 m, 1,0 x 0,80 m, 1x 0,60 m, entre linhas e entre plantas, respectivamente); e, nas subparcelas, 11 épocas de colheita (outubro/2010, novembro/ 2010, dezembro/2010, janeiro/2011, fevereiro/2011, março/ 2011, abril/2011, maio/2011, junho/2011, agosto/2011, setembro/2011) que corresponderam a 213, 247, 270, 300, 334, 368, 398, 430, 460, 521, 550 dias após o transplantio (DAT), respectivamente. Não foram realizadas extrações de óleo essencial no mês de julho, pois as plantas estavam sem folhas. A área de cada parcela (espaçamentos) foi de $132 \mathrm{~m}^{2}(4,0 \mathrm{~m} \times 33,0 \mathrm{~m}) \mathrm{e}$, a das subparcelas (épocas de colheita), de $12 \mathrm{~m}^{2}(3,0 \mathrm{~m} \times 4,0)$ de área útil.

As mudas foram obtidas de propagação vegetativa por estaquia, utilizando-se estacas apicais de $5,0 \mathrm{~cm}$ de comprimento e 2,0 mm de diâmetro, coletadas de plantas matrizes do Horto Medicinal da UTFPR. As estacas foram enraizadas em bandejas de poliestireno, de 72 células, contendo substrato comercial, sob nebulização intermitente.

As plantas, com dez centímetros de altura, foram transplantadas, em 28 de março de 2010, para covas de $0,3 \times 0,3$ $\mathrm{m}$, nos espaçamentos de $1,0 \times 1,0 \mathrm{~m}, 1,0 \times 0,80 \mathrm{~m}, 1 \times 0,60 \mathrm{~m}$, entre linhas e entre plantas, respectivamente. Na adubação de plantio, foram usados $3 \mathrm{~L} /$ cova de cama de aviário fermentada e, na adubação de cobertura, $1 \mathrm{~L} /$ planta, aos 300 dias após o transplantio. Para a fermentação, a cama de aviário foi colocada em leira, em que se realizou revolvimento, para permitir aeração e controle de temperatura, e ali, ficou curtindo por três meses (Kiehl, 2004). Durante o período de cultivo da Aloysia triphylla, foram realizadas irrigações, por microaspersão, e controle de plantas espontâneas, por meio de capinas manuais.

Os dados meteorológicos (precipitação, temperatura, umidade relativa do ar e radiação solar) foram obtidos da estação meteorológica (16 UTC) da UTFPR - Campus Dois
Vizinhos, localizada próxima à unidade experimental (Tabela 1).

O óleo essencial de todas as colheitas (outubro/2010 a setembro/2011 e janeiro/2012) foi extraído por hidrodestilação, em aparelho de Clevenger modificado, por 120 minutos, com três repetições por tratamento. Foram utilizadas amostras compostas de $70 \mathrm{~g}$ de folhas frescas sadias, de três plantas por parcela, colhidas às oito horas da manhã, em cada tratamento. As folhas foram coletadas nas regiões apical, mediana e basal, formando uma amostra representativa da planta, e picadas em fragmentos de aproximadamente $1,0 \mathrm{~cm}$, imediatamente antes da extração. O hidrolato obtido foi submetido à partição líquidolíquido, em funil de separação, com três porções de $25 \mathrm{~mL}$ de diclorometano, por 20 minutos, cada. As frações orgânicas de cada repetição foram reunidas e secadas com uma porção de sulfato de magnésio anidro. O sal foi removido por filtração simples e, o solvente, evaporado, à temperatura ambiente, em capela de exaustão, até alcançar peso constante, obtendo-se, assim, o óleo essencial purificado. Após a obtenção do óleo essencial puro, foi obtida sua massa, sendo, o teor, calculado pela fórmula: $\mathrm{T}(\%)$ = Massa do óleo $(\mathrm{g}) / 70 \mathrm{~g}$ de folhas frescas x 100.

As análises químicas do óleo essencial foram realizadas em sistema de cromatografia gasosa HP5890, equipado com detector de ionização em chamas, com coluna BP1 (SGE) $(25 \mathrm{~m} \times 0,25 \mathrm{~mm} \times 0,25 \mu \mathrm{m})$. Utilizou-se hidrogênio, como gás de arraste $\left(2 \mathrm{~mL} \mathrm{~min}^{-1}\right)$; a temperatura do injetor e do detector foi de $220^{\circ} \mathrm{C}$. A temperatura inicial do forno foi de $60^{\circ} \mathrm{C}$, mantendo-se uma isoterma de $1 \mathrm{~min}$, seguida por uma rampa de temperatura de $3^{\circ} \mathrm{C} / \mathrm{min}$ até $220^{\circ} \mathrm{C}$. O óleo foi diluído em clorofórmio ( $\left.1 \% \mathrm{v} / \mathrm{v}\right)$ e $1 \mu \mathrm{L}$ foi injetado automaticamente no cromatógrafo, no modo split, a uma razão de injeção de 1:50. A identificação dos picos foi realizada por comparação dos índices de retenção, relativos à coinjeção da série linear de $n$-alcanos (C10 a C18), com índices de retenção de literatura (Adams, 2001).

A colheita final foi realizada aos 662 dias após o transplantio das mudas. No mês de janeiro de 2012, no período da manhã, realizou-se a coleta de cinco plantas centrais de cada parcela, para determinação de parâmetros de crescimento (biomassas fresca e seca das folhas, área foliar e altura das plantas), teor e rendimento de óleo essencial, por planta. O rendimento foi calculado pela fórmula $\mathrm{ROE}=$ massa de óleo $(\mathrm{g}) \mathrm{x}$ massa fresca da planta $/ \mathrm{g}$ de folha fresca. A área foliar foi determinada em medidor de área foliar modelo LICOR. A altura foi determinada com auxílio de trena, realizando-se medidas do colo até o ápice da planta.

Os resultados de teor, rendimento e composição química do óleo essencial, biomassas fresca e seca das folhas, área foliar e altura das plantas foram submetidos à análise de variância com auxílio do programa "SAS" (SAS, 
1999). As variâncias dos tratamentos foram testadas, quanto à homogeneidade, pelo teste de Bartlett, e, as médias, comparadas pelo teste de Tukey, a $5 \%$ de probabilidade.

\section{RESULTADOS E DISCUSSÃO}

Não houve interação significativa entre espaçamentos e épocas de colheita (meses), para as variáveis analisadas. Considerando-se as colheitas realizadas nos 11 meses, verificou-se que, no espaçamento $1,0 \times 1,0 \mathrm{~m}$, o teor de óleo essencial foi maior $(0,32 \%)$ que nos demais. $\mathrm{O}$ adensamento de plantas resultou em menor teor de óleo essencial $(0,21 \%)$ (Tabela 2$)$.

Nas condições em que o estudo foi realizado, constatou-se que a distribuição das plantas no espaçamento $1,0 \times 1,0$ m permitiu maior incidência dos raios solares, o que, provavelmente, contribuiu para maior atividade fotossintética, área foliar e produção de biomassa, resultando em maior teor de óleo essencial.

Marco et al. (2007) avaliaram o efeito das densidades de plantio $(0,50 \times 0,50 \mathrm{~m}, 0,50 \times 0,80 \mathrm{~m}$ e $0,80 \times 0,80 \mathrm{~m})$, na produção de óleo essencial de capim-citronela (Cymbopogon winterianum Jowitt.), e obtiveram maior produção de óleo essencial no maior espaçamento avaliado (40 $\mathrm{mL} \mathrm{kg}^{-1}$ de massa seca). Segundo os autores, em espaçamentos maiores, ocorre maior desenvolvimento da planta e da área foliar, contribuindo para aumentar o teor dos princípios ativos na planta.

No mês de fevereiro, observou-se o maior teor de óleo essencial $(0,45 \%)$. Nesse mês, a planta estava em pleno crescimento vegetativo, caracterizado pelo aumento abundante de biomassa, especialmente de folhas jovens. Adzet et al. (1992) verificaram que, para produção de óleos essenciais, folhas jovens são fisiologicamente mais ativas que folhas maduras. Brant et al (2008) observaram comportamento semelhante, com a mesma espécie, na região de Lavras, Minas Gerais.

Os meses de abril, maio e junho apresentaram os menores valores de teor de óleo essencial $(0,16$ a 0,19\%), não diferindo estatisticamente, entre si e do mês de agosto de 2011 (Tabela 2). Nos meses de maio e junho, estação de outono-inverno, verificou-se queda de temperaturas (16,9 e $\left.14,5^{\circ} \mathrm{C}\right)$ e de radiação $\left(596,5\right.$ e $\left.496,8 \mathrm{~kJ} \mathrm{~m}^{-2}\right)$. Nesses meses, também foram observados queda das folhas e crescimento lento das plantas decorrentes dos fatores ambientais adversos. É importante considerar que as plantas de Aloysia triphylla, na região sul do Brasil, iniciam a perda das folhas nos meses de maio e junho, com uma taxa gradativa de $40 \%$, havendo ainda folhas suficientes para extração de óleo essencial. No mês de julho, com a ocorrência de geadas, a taxa de perda de folhas foi de $100 \%$, não houve folhas para extração de óleo essencial. Os re-

Tabela 1. Dados metereológicos de temperatura, radiação, precipitação e umidade relativa da UTFPR - Campus Dois Vizinhos, PR, no período de janeiro de 2010 a dezembro de 2011. Dois Vizinhos, PR

\begin{tabular}{|c|c|c|c|c|}
\hline Data & Temperaturamédia $\left({ }^{\circ} \mathbf{C}\right)$ & $\operatorname{Radiação~}\left(\mathbf{k J} \mathbf{m}^{-2}\right)$ & Precipitação (mm) & Umidade Relativa (\%) \\
\hline Janeiro/10 & 23,9 & 922,9 & 293,2 & 81,5 \\
\hline Fevereiro/10 & 24,4 & 948,3 & 216,0 & 79,6 \\
\hline Março/10 & 23,1 & 893,4 & 178,0 & 74,4 \\
\hline Abril/10 & 20,0 & 717,8 & 198,6 & 74,8 \\
\hline Maio/10 & 16,1 & 634,1 & 127,4 & 83,1 \\
\hline Junho/10 & 16,4 & 810,4 & 60,2 & 76,1 \\
\hline Julho/10 & 16,1 & 766,9 & 76,6 & 77,8 \\
\hline Agosto/10 & 17,0 & 714,8 & 34,8 & 65,7 \\
\hline Setembro/10 & 19,8 & 687,8 & 28,0 & 62,5 \\
\hline Outubro/10 & 19,6 & 864,4 & 260,6 & 67,0 \\
\hline Novembro/10 & 21,0 & 1070,5 & 272,6 & 69,3 \\
\hline Dezembro/10 & 22,2 & 1145,4 & 267,6 & 81,1 \\
\hline Janeiro/11 & 23,6 & 995,1 & 198,8 & 81,7 \\
\hline Fevereiro/11 & 24,3 & 830,1 & 186,0 & 84,8 \\
\hline Março/11 & 23,3 & 886,1 & 70,8 & 72,1 \\
\hline Abril /11 & 20,6 & 806,8 & 104,8 & 76,5 \\
\hline Maio/11 & 16,9 & 596,5 & 31,0 & 76,6 \\
\hline Junho/11 & 14,5 & 496,8 & 142,2 & 78,5 \\
\hline Julho/11 & 15,9 & 499,8 & 263,0 & 76,6 \\
\hline Agosto/11 & 16,5 & 653,5 & 251,0 & 75,4 \\
\hline Setembro/11 & 19,0 & 1003,2 & 66,2 & 64,6 \\
\hline Outubro/11 & 20,9 & 912,1 & 271,8 & 69,6 \\
\hline Novembro/11 & 21,6 & 1020,5 & 139,0 & 67,8 \\
\hline Dezembro/11 & 23,2 & 1179,3 & 36,8 & 65,5 \\
\hline
\end{tabular}


sultados de teor de óleo essencial, nesses meses, sugerem ser decorrentes das condições climáticas (temperatura e radiação). Segundo Sangwan et al. (2001), a radiação é um importante fator que pode interferir na produção e na qualidade dos óleos essenciais, por meio da fotossíntese, da modulação do fotoperíodo e da qualidade da luz.

Nos meses de maio e junho, em função das condições climáticas terem sido desfavoráveis ao crescimento, possivelmente ocorreu ativação do mecanismo natural fontedreno, o qual degrada os metabólitos secundários e os direciona para a manutenção do metabolismo primário (Taiz \& Zeiger, 2004).

Brant et al. (2008) também verificaram redução no teor de óleo essencial de Aloysia triphylla, nos meses de junho $(0,133 \%)$ e agosto $(0,020 \%)$, por causa das condições climáticas adversas (temperatura, precipitação e umidade) e do crescimento lento das plantas. Esse efeito também foi observado por Deschamps et al. (2008), que constataram, a diminuição do teor de óleo essencial, em diferentes espécies de Mentha, por ocasião do inverno, relacionada com a diminuição da temperatura, umidade $\mathrm{e}$ radiação.

Outro fator, que pode ter contribuído para os resultados nos meses de abril a junho, foi o desenvolvimento fisiológico da planta. A Aloysia tryphylla emite folhas em processo continuado, havendo maturação e posterior senescência das folhas mais velhas, que secam ou caem dos ramos nessa estação, diminuindo a biomassa foliar. Isso implica, necessariamente, ajuste de época de colheita antes da senescência das folhas.

A análise cromatográfica dos óleos essenciais, extraídos de plantas nos diferentes espaçamentos, revelou grande variabilidade na composição química. Os constituintes identificados no óleo essencial de Aloysia triphylla foram: geranial (28,39 a 36,90\%), neral (19,17 a 27,55\%), limoneno (11,20 a 16,15\%), nerolidol (5,23 a 12,34\%), além de E- $\beta$-ocimeno, zingibereno, cariofileno, $\beta$-pineno, sabineno, germacreno $\mathrm{D}$, ar-curcumeno, acetato de geranila, terpinen-4ol, $\alpha$-terpineol, linalool, trans óxido limoneno, $\alpha$-pineno, mirceno e cis- oxido- limoneno em menores quantidades (Tabela 3). A mistura dos isômeros (geranial e neral) forma o citral, principal constituinte do óleo essencial de Aloysia triphylla (Lorenzi \& Mattos, 2008).

O espaçamento $1,0 \times 1,0 \mathrm{~m}$ resultou em maior teor de geranial $(36,90 \%)$ e linalol $(1,20 \%)$. Os espaçamentos $1,0 \mathrm{x}$ $1,0 \mathrm{~m}$ e 1,0 x 0,8 m apresentaram os maiores teores de neral (27,55 e $25,53 \%)$ e limoneno (16,15 e 14,24\%), respectivamente. Já os teores de $\alpha$-pineno $(0,53$ a $0,83 \%)$, mirceno $(0,42$ a $0,50 \%)$, cis óxido limoneno $(0,30$ a $0,40 \%)$ não diferiram, estatisticamente, nos espaçamentos avaliados. $\mathrm{O}$ adensamento de plantas alterou a composição qualitativa e quantitativa do óleo essencial, resultando em menores teores de geranial, neral e limoneno (Tabela 3).

Brant et al. (2009) encontraram teores semelhantes de neral $(20 \%)$ e geranial (30\%), em plantas de Aloysia triphylla, cultivadas no espaçamento de 1,0 x 1,0 m.

Nas análises de Brant et al. (2009), o citral (geranial + neral) correspondeu a, aproximadamente, $50 \%$ do óleo essencial. Já, neste trabalho, o teor de citral foi de $64,45 \%$, no espaçamento de 1,0 x 1,0 m.

Nos meses de outubro a março, observaram-se maiores teores do óleo essencial geranial (36,0 a 39,0\%). Os meses de agosto a abril apresentaram os maiores teores de neral (24,50 a 29,70\%). O mês de junho apresentou o menor teor de geranial $(26,17 \%)$ e, os meses de maio e junho, os menores teores de neral (20,30 e 18,04\%), respectivamente (Tabela 4). Nesses meses, as temperaturas $\left(19,6 ; 21,0 ; 22,2 ; 23,6 ; 24,3\right.$ e $\left.23,3^{\circ} \mathrm{C}\right)$, as radiações $(864,4$;

Tabela 2. Teor de óleo essencial de folhas frescas de Aloysia triphylla em resposta de espaçamentos e épocas de colheita. Dois Vizinhos, UTFPR, 2011

\begin{tabular}{lcccc}
\hline Épocas/Espaçamentos & $\mathbf{1 , 0} \mathbf{x} \mathbf{1 , 0} \mathbf{~ m}$ & $\mathbf{1 , 0} \mathbf{x} \mathbf{0 , 8} \mathbf{m}$ & $\mathbf{1 , 0} \mathbf{x} \mathbf{0 , 6} \mathbf{m}$ & Média: mês \\
\hline Outubro/2010 & 0,39 & 0,30 & 0,26 & $0,32 \mathrm{bc}^{*}$ \\
Novembro/2010 & 0,33 & 0,27 & 0,20 & $0,27 \mathrm{~cd}$ \\
Dezembro/2010 & 0,34 & 0,31 & 0,27 & $0,31 \mathrm{c}$ \\
Janeiro/2011 & 0,45 & 0,37 & 0,31 & $0,38 \mathrm{~b}$ \\
Fevereiro/2011 & 0,55 & 0,42 & 0,37 & $0,28 \mathrm{c}$ \\
Março/2011 & 0,33 & 0,28 & 0,22 & $0,19 \mathrm{e}$ \\
Abril/2011 & 0,24 & 0,17 & 0,15 & $0,16 \mathrm{e}$ \\
Maio/2011 & 0,17 & 0,16 & 0,14 & $0,17 \mathrm{e}$ \\
Junho/2011 & 0,21 & 0,19 & 0,10 & $0,21 \mathrm{de}$ \\
Agosto/2011 & 0,24 & 0,21 & 0,17 & $0,26 \mathrm{~cd}$ \\
Setembro/2011 & 0,33 & 0,24 & 0,20 & \\
Média: espaçamento & $0,32 \mathrm{~A}$ & $0,26 \mathrm{~B}$ & $0,21 \mathrm{C}$ & \\
\hline CV $(\%)$ & & &
\end{tabular}

* Médias seguidas da mesma letra, maiúscula na linha e minúscula na coluna, não diferem estatisticamente pelo teste de Tukey, a 5\% de probabilidade.

Rev. Ceres, Viçosa, v. 60, n.3, p. 372-379, mai/jun, 2013 
1070,$5 ; 1145,4 ; 995,1 ; 830,1$ e $886,1 \mathrm{~kJ} \mathrm{~m}^{-2}$ ) e as precipitações $(260,6 ; 272,6 ; 267,6 ; 198,8 ; 186$ e 70,8 mm), respectivamente, foram mais elevadas e, nos meses de maio e junho, houve diminuição nas temperaturas médias $(16,9 \mathrm{e}$ $\left.14,5^{\circ} \mathrm{C}\right)$, nas radiações $\left(596,5\right.$ e $\left.496,8 \mathrm{~kJ} \mathrm{~m}^{-2}\right)$ e nas precipitações (31 e 142,2 mm), respectivamente.

A importância comercial do óleo essencial da Aloysia triphylla depende da percentagem de geranial e neral, bem como da baixa percentagem de outros componentes indesejáveis, tais como o nerol e geraniol, que são formas oxidativas do neral e geranial, e que afetam a sua qualidade (Tabatavaie \& Nazari, 2007). Esses componentes oxidativos (nerol e geraniol) não foram encontrados no óleo essencial.

Com relação à altura, à área foliar, às biomassas fresca e seca, ao teor e ao rendimento de óleo essencial, observaram-se maiores valores no espaçamento 1,0 x 1,0 m (Tabela 5 ), em que as plantas estavam mais ramificadas, em relação às dos espaçamentos menores. A menor densidade permitiu uma distribuição mais uniforme das plantas por área, possibilitando a utilização mais eficiente de luz, água e nutrientes, o que pode ter contribuído para o resultado obtido. Os dados de produção de biomassa são muito importantes para o produtor, pois, além do óleo essencial, as folhas de A. triphylla são comercializadas para chás.

A maior área foliar no espaçamento $1,0 \times 1,0 \mathrm{~m}$ pode ser devida à maior exposição das folhas à luz difusa, resultando no aumento da área foliar específica, pela maior expansão foliar e maior acúmulo de fotoassimilados, o que contribui para maior produção de biomassa (Favorito et al., 2011).

Apesar de o espaçamento 1,0 x 0,6 m apresentar maior número de plantas por área (16.667 plantas ha-1), ele não superou a produção de biomassa fresca $\left(12.660 \mathrm{~kg} \mathrm{ha}^{-1}\right) \mathrm{e}$ seca $\left(5.940 \mathrm{~kg} \mathrm{ha}^{-1}\right)$, obtida no espaçamento 1,0 x 1,0 m. Constatou-se que, para essa espécie, por apresentar porte arbustivo e ramificado, o adensamento de plantas exerceu influência na arquitetura, no crescimento, no teor e no rendimento de óleo essencial.

Segundo Minami et al. (1998), o aumento da densidade populacional resulta na competição entre plantas, sendo o desenvolvimento individual prejudicado. De acordo com Biasi \& Deshamps (2009), os menores espaçamentos resultam em menor aproveitamento dos recursos do meio, não expressando todo o potencial genético da planta.

Resultados semelhantes foram observados por Monteiro et al. (2011), em Mentha campestris Schur, quando os autores constataram que, na segunda época de colheita, quando as plantas apresentavam desenvolvimento superior, o plantio mais adensado e a consequente maior redução de radiação disponível no dossel das plantas, reduziu o teor de óleo essencial. Marco et al. (2007) observaram que capim-citronela, em espaçamentos mais largos $(0,80 \times 0,80 \mathrm{~m})$, apresentou produção de óleo essencial superior à dos espaçamentos 0,50 x 0,80 m e 0,50 x 0,50 $\mathrm{m}$, em plantas cortadas a $15 \mathrm{~cm}$ do solo.

Tabela 3. Componentes do óleo essencial (\%) de Aloysia triphylla em resposta a espaçamentos. Dois Vizinhos, UTFPR, 2011

\begin{tabular}{|c|c|c|c|c|}
\hline \multirow{2}{*}{ Componentes } & \multirow{2}{*}{$* \mathbf{I K}$} & \multicolumn{3}{|c|}{ Espaçamentos } \\
\hline & & $1,0 \times 1,0 \mathrm{~m}$ & $1,0 \times 0,8 \mathrm{~m}$ & $1,0 \times 0,6 \mathrm{~m}$ \\
\hline$\alpha$-pineno & 960 & $0,53 \mathrm{a} * *$ & $0,83 \mathrm{a}$ & $0,64 a$ \\
\hline Sabineno & 980 & $0,34 b$ & $0,50 b$ & $1,75 \mathrm{a}$ \\
\hline$\beta$-pineno & 982 & $0,68 b$ & $1,51 \mathrm{a}$ & $0,90 b$ \\
\hline Mirceno & 994 & $0,42 \mathrm{a}$ & $0,50 \mathrm{a}$ & $0,45 \mathrm{a}$ \\
\hline Limoneno & 1020 & $16,15 \mathrm{a}$ & $14,24 \mathrm{a}$ & $11,20 \mathrm{~b}$ \\
\hline (E)- $\beta$-ocimeno & 1036 & $0,41 \mathrm{c}$ & $0,96 b$ & $2,31 \mathrm{a}$ \\
\hline Linalol & 1078 & $1,20 \mathrm{a}$ & $0,90 b$ & $0,85 b$ \\
\hline Cis óxido limoneno & 1103 & $0,30 \mathrm{a}$ & $0,33 \mathrm{a}$ & $0,40 \mathrm{a}$ \\
\hline Trans - oxido limoneno & 1113 & $0,53 \mathrm{c}$ & $1,29 b$ & $1,49 \mathrm{a}$ \\
\hline Terpinen-4ol & 1149 & $0,75 b$ & $1,27 \mathrm{a}$ & $1,63 \mathrm{a}$ \\
\hline$\alpha$-terpineol & 1162 & $0,22 b$ & $1,06 \mathrm{a}$ & $1,40 \mathrm{a}$ \\
\hline Neral & 1208 & $27,55 \mathrm{a}$ & $25,53 a$ & $19,17 b$ \\
\hline Geranial & 1239 & $36,90 \mathrm{a}$ & $29,10 b$ & $28,39 b$ \\
\hline Acetato de geranila & 1363 & $2,00 \mathrm{~b}$ & $3,08 \mathrm{a}$ & $2,52 b$ \\
\hline Cariofileno & 1405 & $0,70 \mathrm{~b}$ & $2,57 \mathrm{a}$ & $2,66 \mathrm{a}$ \\
\hline Germacreno D & 1464 & $0,98 b$ & $1,85 \mathrm{a}$ & $1,40 \mathrm{a}$ \\
\hline Ar-curcumeno & 1468 & $1,30 \mathrm{c}$ & $5,30 \mathrm{a}$ & $2,40 \mathrm{~b}$ \\
\hline Zingibereno & 1483 & $0,95 \mathrm{c}$ & $1,45 b$ & $2,11 \mathrm{a}$ \\
\hline Nerolidol & 1558 & $6,89 b$ & $5,23 \mathrm{~b}$ & $12,34 \mathrm{a}$ \\
\hline Total identificado & & 98,8 & 97,5 & 94,01 \\
\hline
\end{tabular}

* IK=Índice de Kovats

**Médias seguidas da mesma letra na linha não diferem estatisticamente pelo teste de Tukey, a 5\% de probabilidade. 


\begin{tabular}{|c|c|c|c|c|c|c|c|c|c|c|c|c|}
\hline \multirow{3}{*}{ Compostos } & \multirow{3}{*}{$* \mathbf{I K}$} & \multicolumn{11}{|c|}{ Meses } \\
\hline & & \multicolumn{3}{|c|}{2010} & \multicolumn{8}{|c|}{2011} \\
\hline & & $\mathbf{O}$ & $\mathbf{N}$ & $\mathbf{D}$ & $\mathbf{J}$ & $\mathbf{F}$ & $\mathbf{M}$ & $\mathbf{A}$ & $\mathbf{M}$ & $\mathbf{J}$ & A. & $\mathbf{S}$ \\
\hline$\alpha$-pineno & 960 & $1,01 a^{* *}$ & $0,85 \mathrm{a}$ & - & - & - & $0,61 \mathrm{a}$ & $0,75 a$ & $0,55 \mathrm{a}$ & $0,90 \mathrm{a}$ & $0,70 \mathrm{a}$ & $0,80 \mathrm{a}$ \\
\hline Sabineno & 980 & $1,69 \mathrm{a}$ & $0,33 b$ & - & - & - & $0,32 b$ & $0,30 \mathrm{~b}$ & $0,52 b$ & $1,77 \mathrm{a}$ & - & $1,75 \mathrm{a}$ \\
\hline$\beta$-pineno & 982 & $1,65 \mathrm{a}$ & $0,70 \mathrm{~b}$ & $0,71 b$ & - & $0,60 \mathrm{~b}$ & $0,72 b$ & $0,85 b$ & $0,80 \mathrm{~b}$ & $1,40 \mathrm{a}$ & $0,87 \mathrm{~b}$ & $1,52 \mathrm{a}$ \\
\hline Mirceno & 994 & $0,40 \mathrm{a}$ & - & - & - & - & $0,50 \mathrm{a}$ & $0,41 \mathrm{a}$ & $0,49 \mathrm{a}$ & $0,47 \mathrm{a}$ & $0,40 \mathrm{a}$ & $0,40 \mathrm{a}$ \\
\hline Limoneno & 1020 & $13,60 \mathrm{~b}$ & $14,63 \mathrm{a}$ & $16,10 \mathrm{a}$ & $15,26 \mathrm{a}$ & $17,20 \mathrm{a}$ & $10,17 b$ & $12,00 \mathrm{~b}$ & $14,10 \mathrm{a}$ & $12,80 \mathrm{~b}$ & $9,00 \mathrm{c}$ & $13,40 \mathrm{~b}$ \\
\hline (E)- $\beta$-ocimeno & 1036 & $0,74 \mathrm{~b}$ & $0,60 \mathrm{c}$ & $0,80 \mathrm{~b}$ & $0,40 \mathrm{c}$ & $0,42 \mathrm{c}$ & $0,90 \mathrm{~b}$ & 0,55 & $2,20 \mathrm{a}$ & $2,40 \mathrm{a}$ & $2,30 \mathrm{a}$ & $1,40 \mathrm{~b}$ \\
\hline Linalol & 1078 & $1,20 \mathrm{a}$ & $0,85 b$ & $0,90 \mathrm{~b}$ & $1,02 \mathrm{a}$ & $1,10 \mathrm{a}$ & $1,07 \mathrm{a}$ & $0,73 b$ & $0,75 b$ & $0,83 b$ & $0,80 \mathrm{~b}$ & $0,53 \mathrm{c}$ \\
\hline Cis óxido limoneno & 1103 & $0,39 a$ & $0,34 \mathrm{a}$ & $0,30 \mathrm{a}$ & $0,35 \mathrm{a}$ & $0,27 \mathrm{a}$ & $0,29 a$ & $0,31 \mathrm{a}$ & $0,30 \mathrm{a}$ & $0,40 \mathrm{a}$ & $0,29 a$ & $0,37 \mathrm{a}$ \\
\hline Trans-oxido limoneno & 1113 & $1,30 \mathrm{a}$ & $0,91 \mathrm{~b}$ & $0,80 \mathrm{~b}$ & $1,20 \mathrm{a}$ & $0,44 \mathrm{c}$ & $0,60 \mathrm{~b}$ & $0,50 \mathrm{c}$ & $1,30 \mathrm{a}$ & $1,20 \mathrm{a}$ & $1,29 \mathrm{a}$ & $1,45 \mathrm{a}$ \\
\hline Terpinen-4ol & 1149 & $0,70 \mathrm{~b}$ & $0,71 b$ & $1,25 \mathrm{a}$ & $0,79 b$ & $0,75 b$ & $0,80 \mathrm{~b}$ & $0,78 \mathrm{~b}$ & $1,26 \mathrm{a}$ & $1,50 \mathrm{a}$ & $1,49 \mathrm{a}$ & $1,65 \mathrm{a}$ \\
\hline$\alpha$-terpineol & 1162 & $0,23 \mathrm{c}$ & $0,40 \mathrm{~b}$ & - & $0,38 b$ & $0,30 \mathrm{~b}$ & $1,33 \mathrm{a}$ & $1,38 \mathrm{a}$ & $1,38 \mathrm{a}$ & $1,10 \mathrm{a}$ & $0,50 b$ & $0,27 \mathrm{c}$ \\
\hline Neral & 1208 & $24,50 \mathrm{a}$ & $25,28 \mathrm{a}$ & $26,43 \mathrm{a}$ & $27,45 \mathrm{a}$ & $29,70 \mathrm{a}$ & $28,30 \mathrm{a}$ & $26,13 \mathrm{a}$ & $20,30 b$ & $18,02 b$ & $29,31 \mathrm{a}$ & $29,04 \mathrm{a}$ \\
\hline Geranial & 1239 & $36,00 \mathrm{a}$ & $37,12 \mathrm{a}$ & $36,89 a$ & $36,40 \mathrm{a}$ & $39,00 \mathrm{a}$ & $36,30 \mathrm{a}$ & $33,20 \mathrm{~b}$ & $29,39 b$ & $26,17 \mathrm{c}$ & $31,11 \mathrm{~b}$ & $33,55 b$ \\
\hline Acetato de geranila & 1363 & $2,15 b$ & $2,10 \mathrm{~b}$ & $2,74 \mathrm{ab}$ & $3,02 \mathrm{a}$ & $2,60 \mathrm{~b}$ & $3,10 \mathrm{a}$ & $2,30 \mathrm{~b}$ & $2,04 b$ & $3,00 \mathrm{a}$ & $2,40 \mathrm{~b}$ & $2,10 \mathrm{~b}$ \\
\hline Cariofileno & 1405 & $2,50 \mathrm{a}$ & $2,60 \mathrm{a}$ & $2,58 \mathrm{a}$ & $0,75 \mathrm{c}$ & - & $1,80 \mathrm{~b}$ & $1,73 b$ & $2,70 \mathrm{a}$ & $2,40 \mathrm{a}$ & $0,78 \mathrm{c}$ & $2,30 \mathrm{a}$ \\
\hline Germacreno D & 1464 & - & $0,90 \mathrm{~b}$ & - & $1,70 \mathrm{a}$ & $0,85 b$ & $0,80 \mathrm{~b}$ & $1,74 \mathrm{a}$ & $1,90 \mathrm{a}$ & $1,75 \mathrm{a}$ & $1,72 \mathrm{a}$ & - \\
\hline Ar-curcumeno & 1468 & $2,10 \mathrm{~b}$ & $1,80 \mathrm{~b}$ & $1,65 b$ & $1,20 \mathrm{c}$ & - & $1,00 \mathrm{c}$ & $4,80 \mathrm{a}$ & $6,24 a$ & 1,96 & $2,50 \mathrm{~b}$ & $1,90 \mathrm{~b}$ \\
\hline Zingibereno & 1483 & $1,00 \mathrm{~b}$ & $1,09 \mathrm{~b}$ & $0,80 \mathrm{~b}$ & $0,19 \mathrm{c}$ & $0,85 b$ & $2,10 \mathrm{a}$ & $2,00 \mathrm{a}$ & $2,05 \mathrm{a}$ & $1,40 \mathrm{~b}$ & $2,20 \mathrm{a}$ & $1,14 \mathrm{~b}$ \\
\hline Nerolidol & 1558 & $4,55 c$ & $5,65 \mathrm{~b}$ & $6,36 \mathrm{~b}$ & $7,34 b$ & $4,78 \mathrm{c}$ & $5,14 \mathrm{~b}$ & $6,10 \mathrm{~b}$ & $6,78 b$ & $13,90 \mathrm{a}$ & $6,58 b$ & $3,80 \mathrm{c}$ \\
\hline Total identificado & & 95,71 & 96,86 & 98,31 & 97,45 & 98,86 & 95,85 & 96,56 & 95,05 & 93,37 & 94,24 & 97,37 \\
\hline
\end{tabular}

* IK=Índice de Kovats e - = Não detectado

***ádias seguidas da mesma letra na linha não diferem estatisticamente pelo teste de Tukey, a 5\% de probabilidade. 
Tabela 5. Altura (Alt.), área foliar (AF), biomassa fresca (BF) e seca (BS), teor de óleo essencial (TOE), rendimento do óleo essencial (REO) de Aloysia triphylla colhida, em janeiro de 2012, aos 662 dias após o transplantio em função de espaçamentos. Dois Vizinhos, UTFPR, 2012

\begin{tabular}{lcccccc}
\hline Espaçamentos & Alt. $(\mathbf{m})$ & $\mathbf{A F}\left(\mathbf{c m}^{2}\right)$ & $\mathbf{B F}\left(\mathbf{g ~ p l a n t a}^{-1}\right)$ & $\mathbf{B S}\left(\mathbf{g ~ p l a n t a}^{-1}\right)$ & TOE$(\%)$ & ROE $(\mathbf{g}$ planta \\
\hline $1,0 \times 1,0 \mathrm{~m}$ & $1,57 \mathrm{a}^{*}$ & $4.460,3 \mathrm{a}$ & $1.266 \mathrm{a}$ & $594 \mathrm{a}$ & $0,30 \mathrm{a}$ & $3,80 \mathrm{a}$ \\
$1,0 \times 0,8 \mathrm{~m}$ & $1,12 \mathrm{~b}$ & $4.112,2 \mathrm{~b}$ & $812 \mathrm{~b}$ & $365 \mathrm{~b}$ & $0,25 \mathrm{~b}$ & $2,03 \mathrm{~b}$ \\
$1,0 \times 0,6 \mathrm{~m}$ & $0,64 \mathrm{c}$ & $4.085,7 \mathrm{~b}$ & $434 \mathrm{c}$ & $168 \mathrm{c}$ & $0,21 \mathrm{c}$ & $0,91 \mathrm{c}$ \\
\hline CV $(\%)$ & 12 & 10 & 22 & 18 & 20 & 13 \\
\hline
\end{tabular}

*Médias seguidas da mesma letra na coluna não diferem estatisticamente pelo teste de Tukey, a 5\% de probabilidade.

\section{CONCLUSÃO}

O espaçamento mais indicado para se obter maior produção de biomassas fresca e seca, teor e rendimento de óleo essencial, em Alloysia triphylla, é 1,0 x 1,0 m.

A época de colheita indicada para se obter maior teor de óleo essencial é fevereiro.

Os componentes químicos do óleo essencial variam com espaçamentos e épocas de colheita, sendo maior o teor de citral no espaçamento 1,0 x 1,0 m e nos meses de setembro a abril.

\section{REFERÊNCIAS}

Adams RP (2001) Identification of essential oil components by gas chromatography/mass spectroscopy. Carol Stream, Allured Publishing. 469p.

Adzet T, Ponz R, Wolf E \& Schulte E (1992) Content and composition of Melissa officinalis oil in relation to leaf position and harvest time. Planta Medica, 58:558-561.

Biasi LA \& Deschamps C (2009) Do cultivo à produção de óleo essencial. In: Sommer, PG (Eds.) Manual de plantas aromáticas. Curitiba, Layer Studio Gráfico e Editora Ltda. p.100-103.

Bhering SB, Santos HG dos, Bognola IA, Cúrcio GR, Manzatto CV, Carvalho Junior W de, Chagas CS, Áglio MLD \& Souza JS (2008) Mapa de solos do Estado do Paraná: legenda atualizada. Rio de Janeiro, Embrapa/IAPAR. 74p.

Brant RS, Pinto JEBP, Bertolucci SKV \& Albuquerque CJB (2008) Teor do óleo essencial de cidrão [Aloysia triphylla (L’ Hér) Britton] em função da variação sazonal. Revista Brasileira de Plantas Medicinais, 10:83-88.

Brant RS, Pinto JEBP, Bertolucci SKV, Silva A \& Albuquerque CJB (2009) Teor do óleo essencial de cidrão [Aloysia triphylla ( $\mathrm{L}^{\prime}$ Hér) Britton] em diferentes horários de colheita e processamento pós-colheita. Ciëncia Agrotecnolgia, 33:2065-2068.

Czepak MP \& Cruciol CAC (2003) Produtividade e composição do óleo essencial de capim-limão (Cymbopogon citratus (DC.) STAPF) em diferentes arranjos espaciais. In: Simpósio Brasileiro de Plantas Medicinais: Diagnóstico e Perspectivas, Campinas. Anais, Instituto Agronômico. p.113 -115.

Deschamps C, Zanatta JL, Bizzo HR, Oliveira MC \& Roswalka LC (2008) Avaliação sazonal do rendimento de óleo essencial em espécies de menta. Ciência Agrotecnologia, 32:725-730.

Favorito PA, Echer MM, Offemann LC, Schlindwein MD, Colombare LF, Schneider RP \& Hachmann TL (2011) Características produtivas do manjericão (Ocimum basilicum L.) em função do espaçamento entre plantas e entre linhas. Revista Brasileira de Plantas Medicinais, 13:582-586.
Figueiredo RO de, Stefanini MB, Ming LC, Marques MOM \& Facanali R (2004) Essential oil composition of Aloysia triphylla (L'Herit) Britton leaves cultivated in Botucatu, São Paulo, Brazil. Acta Horticulturae, 629:131-134.

Kiehl EJ. Manual de compostagen: maturação e qualidade do composto. 2004. Piracicaba, ESALQ. 173p.

Lima HRP, Kaplan MAR \& Cruz AVM (2003) Influência dos fatores abióticos na produção e variabilidade de terpenóides em plantas. Revista Floresta e Ambiente, 10:71-77.

Lorenzi H \& Matos FJA (2008) Plantas medicinais no Brasil: nativas e exóticas. 2.ed. Nova Odessa, Plantarum. 486p.

Marco C, Innecco R, Mattos SH, Borges NSS \& Nagao EO (2007) Características do óleo essencial de capim-citronela em função de espaçamento, altura e época de corte. Horticultura Brasileira, 25:429-432.

Melo MTP, Carvalho Júnior WGO, Souza MF, Figueiredo LS \& Martins ER (2011) Produção de fitomassa e teor de óleo essencial de folhas de alecrim-pimenta (Lippia sidoides Cham.) em diferentes espaçamentos de plantio. Revista Brasileira de Plantas Medicinais, 13:230-234.

Minami K, Cardoso AII, Costa F \& Duarte FR (1998) Efeito do espaçamento sobre a produção em rabanete. Bragantia, 57:169173.

Monteiro R, Deschamps C, Biasi LA \& Bizzo H (2011) Desenvolvimento vegetativo de Mentha campestris Schur e produção de mentol em diferentes espaçamentos de plantio e épocas de colheita. Revista Brasileira de Plantas Medicinais, 13:401-407.

Sangwan NS, Farooqi AHA, Sfiabih F \& Sangwan RS (2001) Regulation of essential oil production in plants. Plant Growth Regulation, 34:3-21.

SAS Institute Inc. (1999) SAS: user's guide statistics: version 8.0 edition.

Tabatavaie SJ \& Nazari J (2007) Influence of nutrient concentrations and $\mathrm{NaCl}$ salinity on the growth, photosynthesis and essential oil content of peppermint and lemon verbena. Turkish Journal of Agriculture and Forestry, 31:245-253.

Taiz L \& Zeiger E (2004) Fisiologia Vegetal. Porto Alegre, Artmed. $719 \mathrm{p}$.

Taveira FSN, Lima WN, Andrade EHA \& Maia JGS (2003) Seasonal essential oil variation of Aniba canelilla. Biochemical Systematics and Ecology, 31:69-75.

Ventrella MC \& Ming LC (2000) Produção de matéria seca e óleo essencial em folhas de erva cidreira sob diferentes níveis de sombreamento e épocas de colheita. Horticultura Brasileira, 18:972-974.

Yadava AK (2001) Cultivation of lemon grass (Cymbopogon flexuosus, 'CKP-25') under Poplar based agroforestry system. Indian Forester, 127:213-223.

Rev. Ceres, Viçosa, v. 60, n.3, p. 372-379, mai/jun, 2013 